\title{
Dense Arbitrarily Vertex Decomposable Graphs
}

\author{
Mirko Horňák • Antoni Marczyk • \\ Ingo Schiermeyer • Mariusz Woźniak
}

Received: 4 January 2011 / Revised: 20 July 2011 / Published online: 8 September 2011

(C) The Author(s) 2011. This article is published with open access at Springerlink.com

\begin{abstract}
A graph $G$ of order $n$ is said to be arbitrarily vertex decomposable if for each sequence $\left(n_{1}, \ldots, n_{k}\right)$ of positive integers such that $n_{1}+\cdots+n_{k}=n$ there exists a partition $\left(V_{1}, \ldots, V_{k}\right)$ of the vertex set of $G$ such that for each $i \in\{1, \ldots, k\}$, $V_{i}$ induces a connected subgraph of $G$ on $n_{i}$ vertices. The main result of the paper reads as follows. Suppose that $G$ is a connected graph on $n \geq 20$ vertices that admits a perfect matching or a matching omitting exactly one vertex. If the degree sum of any pair of nonadjacent vertices is at least $n-5$, then $G$ is arbitrarily vertex decomposable. We also describe 2-connected arbitrarily vertex decomposable graphs that satisfy a similar degree sum condition.
\end{abstract}

Keywords Arbitrarily vertex decomposable graph · Traceable graph · Independence number · Perfect matching

Mathematics Subject Classification (2000) $\quad 05 \mathrm{C} 38 \cdot 05 \mathrm{C} 70$

The work of M. Horňák was supported by Science and Technology Assistance Agency under the contract No. APVT-20-004104 and by Grant VEGA 1/3004/06.

Research financially supported in parts by DAAD contracts TU Freiberg-AGH Krakau and TU

Freiberg-UPJŠ Košice.

M. Horňák

Institute of Mathematics, P.J. Šafárik University, Jesenná 5,

04001 Košice, Slovakia

A. Marczyk $(\varangle) \cdot$ M. Woźniak

AGH University of Science and Technology, Faculty of Applied Mathematics,

Al. Mickiewicza 30, 30059 Kraków, Poland

e-mail: marczyk@agh.edu.pl

I. Schiermeyer

Fakultät für Mathematik und Informatik, Technische Universität Bergakademie Freiberg,

09596 Freiberg, Germany 


\section{Introduction}

Let $G=(V, E)$ be a simple, undirected graph of order $n$. A sequence $\tau=\left(n_{1}, \ldots, n_{k}\right)$ of positive integers is called admissible for $G$ if $n_{1}+\cdots+n_{k}=n$. Such a sequence is said to be realizable in $G$ if there exists a partition $\left(V_{1}, \ldots, V_{k}\right)$ of $V$ such that for each $i \in\{1, \ldots, k\},\left|V_{i}\right|=n_{i}$ and the subgraph induced by $V_{i}$ is connected. This partition of $V$ is called a realization of $\tau$ in $G$. A graph $G$ is arbitrarily vertex decomposable (avd for short) if for each admissible sequence $\tau$ for $G$ there exists a realization of $\tau$ in $G$. Similarly, $G$ is $r$-vertex decomposable if each sequence $\left(n_{1}, \ldots, n_{r}\right)$ of length $r$ admissible for $G$ is realizable in $G$.

Avd graphs have been a subject of interest of several authors in recent years. The most important result on avd trees due to Barth and Fournier [2] (conjectured by Horňák and Woźniak [8]) states that if $T$ is a tree with maximum degree $\Delta(T)$ at least five, then $T$ is not avd.

There are various results characterizing different families of avd trees $[1,2,5,7,8]$. The complete characterization of on-line avd trees was recently found by Horňák et al. [9]. In Kalinowski et al. [10] investigated unicyclic avd graphs where the unique cycle is dominating. They solved also the on-line version of the same problem in [11].

Lovász [13] using a homology theory proved that every $k$-connected graph is $k$-vertex decomposable. This result was independently obtained by Gyóri [6].

Since each traceable graph (i.e., containing a hamiltonian path) is (as is easily seen) avd, each condition implying the existence of a hamiltonian path in a graph also implies that the graph is avd. However, one can ask the question: What happens if we weaken some known condition for traceability? Are the graphs satisfying this weaker condition still avd? In this article we focus on an Ore-type condition for hamiltonicity. Namely, for a graph $G$ of order $n$ define $\sigma_{2}(G):=\min \{d(x)+d(y) \mid x y \notin E\}$ if $G$ is not a complete graph, and $\sigma_{2}(G)=\infty$, otherwise. Ore's well-known theorem [16] implies that if $\sigma_{2}(G) \geq n-1$ then $G$ is traceable, so also avd.

The purpose of this paper is to show that every connected graph $G$ of order $n \geq 20$ with $\sigma_{2}(G) \geq n-5$ is avd if and only if it admits a perfect matching or a quasiperfect matching (a matching omitting exactly one vertex). This theorem (Theorem 14 in Sect. 7) is an extension of a result due to the second author (cf. [14]). We also characterize 2-connected avd graphs $G$ with $\sigma_{2}(G) \geq n-4$.

The problem of deciding whether a given graph is arbitrarily vertex decomposable is NP-complete [1] but we do not know if this problem is NP-complete when restricted to trees. Note also that one can find in [8] some references concerning arbitrarily edge decomposable graphs.

\section{Terminology and Notation}

Let $T=(V, E)$ be a tree. A vertex $x \in V$ is called primary if $d(x) \geq 3$. A graph is a star-like tree if it is a tree homeomorphic to a star $K_{1, q}$ for some $q \geq 3$. Such a tree $T$ consists of $q$ paths $A_{1}, \ldots, A_{q}$ starting at the unique primary vertex of $T$. For each $A_{i}$ let $a_{i} \geq 2$ be the order of $A_{i}$. We shall denote the above defined star-like tree by $S\left(a_{1}, \ldots, a_{q}\right)$. Notice that the order of this star-like tree is equal to $1+\sum_{i=1}^{q}\left(a_{i}-1\right)$. 

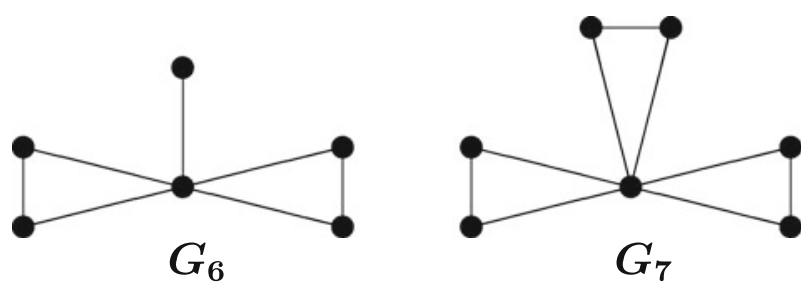

Fig. 1 The graphs $G_{6}$ and $G_{7}$

Let $G$ be a graph and let $C$ be a cycle of $G$ with a given orientation. Suppose $a$ is a vertex of $C$. We shall denote by $a^{+}=a^{+1}$ the successor and by $a^{-}=a^{-1}$ the predecessor of $a$ on $C$. We write $a^{+2}$ for $\left(a^{+}\right)^{+}, a^{-2}$ for $\left(a^{-}\right)^{-}, a^{+k}$ for $\left(a^{+(k-1)}\right)^{+}$, $a^{-k}$ for $\left(a^{-(k-1)}\right)^{-}$and $a^{+0}$ for $a$. If $A$ is a subset of $V(C)$, then $A^{+}=\left\{v^{+} \mid v \in A\right\}$ and $A^{-}=\left\{v^{-} \mid v \in A\right\}$.

Throughout the paper indices of a cycle $C=x_{1}, x_{2}, \ldots, x_{p}, x_{1}$ are to be taken modulo $p$ (i.e., $x_{i}=x_{j}$ whenever $\left.i \equiv j(\bmod p), i, j \in \mathbb{Z}\right)$. If $x \notin V(C)$, we write $N_{C}(x)$ for the set of neighbors of $x$ on $C$ and $d_{C}(x)$ for the cardinality of $N_{C}(x)$.

Let $P=x_{1}, \ldots, x_{r}$ be a path and let $a, b \in V(P)$. By $[a, b]$ we denote the set of consecutive vertices of $P$ from $a$ to $b$ ( $a$ and $b$ included) in the direction specified by the natural orientation of $P$ (from $x_{1}$ to $x_{r}$ ); it is called a segment of $P$ from a to $b$ or simply a segment. We define the symbols $a^{+k}, a^{-k}, N_{P}(x)$ and $d_{P}(x)$ analogously as for a cycle with a given orientation.

A sun with $r$ rays is a graph of order $n \geq 2 r$ with $r$ hanging vertices $u_{1}, \ldots, u_{r}$ whose deletion yields a cycle $C_{n-r}$, and each vertex $v_{i}$ (on $C_{n-r}$ ) adjacent to $u_{i}$ is of degree three. If the vertices $v_{1}, \ldots, v_{r}$ are situated on the cycle $C_{n-r}$ in such a way that there are exactly $b_{i} \geq 0$ vertices, each of degree two, between $v_{i}$ and $v_{i+1}$ $, i=1, \ldots, r$, then this sun is denoted by $\operatorname{Sun}\left(b_{1}, \ldots, b_{r}\right)$, and is unique up to isomorphism. Clearly, every sun with one ray is avd since it is traceable.

The join of two vertex-disjoint graphs $G$ and $H$ is the graph denoted by $G \vee H$ obtained from $G \cup H$ by adding all edges between $V(G)$ and $V(H)$.

For terminology and concepts not defined here we refer the reader to [4].

\section{Two Families of Non-avd Graphs}

Let $n \geq 4$ be an integer. Consider the disjoint union $K_{p} \cup K_{q} \cup K_{r}$ of three complete graphs such that $p+q+r=n-1$ and denote by $G_{n}$ the join $K_{1} \vee\left(K_{p} \cup K_{q} \cup K_{r}\right)$. We will assume that $p \leq q \leq r \leq p+1$, hence $n-1=3 p+d$, where $0 \leq d \leq 2$ ( $G_{6}$ and $G_{7}$ are shown in Fig. 1).

Observe that $\sigma_{2}\left(G_{n}\right)=\left\lfloor\frac{2 n}{3}\right\rfloor$ for $n \equiv 1(\bmod 3)$ and $\sigma_{2}\left(G_{n}\right)=\left\lfloor\frac{2 n}{3}\right\rfloor-1$ otherwise. Every connected subgraph of the graph $K_{p} \cup K_{q} \cup K_{r}$ contains at most $p+1$ vertices and the integer $w=n-2(p+2)=p+d-3$ is non-negative for $p \geq 3$. This implies that the sequence $(w, p+2, p+2)((p+2, p+2)$ for $w=0)$ is admissible and non-realizable in $G_{n}$ for $n \geq 10$. It is easy to check that for each $4 \leq n \leq 9, n \neq 5$ $(p \leq 2)$ there is an admissible sequence of the form $(w, p+2, p+2),(p+2, p+2)$, $(w, p+1, p+1)$ or $(p+1, p+1)$ which is not realizable in the graph $G_{n}$. 

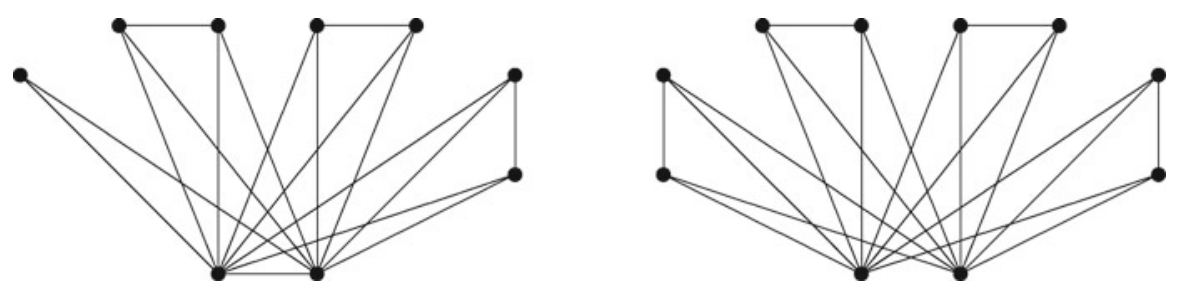

Fig. 2 The graphs $H_{9}$ and $H_{10}^{\prime}$

Similarly, for $n \geq 6$ let $H_{n}$ ( $H_{n}^{\prime}$, resp.) denote the join $K_{2} \vee\left(K_{p} \cup K_{q} \cup K_{r} \cup K_{s}\right)$ $\left(2 K_{1} \vee\left(K_{p} \cup K_{q} \cup K_{r} \cup K_{s}\right)\right.$, resp.), where $p+q+r+s=n-2$ and the numbers $p, q, r, s$ are as equal as possible (the graphs $H_{9}$ and $H_{10}^{\prime}$ are illustrated in Fig. 2). Hence, $H_{n}$ can be obtained from $H_{n}^{\prime}$ by deleting the edge connecting two vertices of degree $n-1$ in $H_{n}$. Thus, $p \leq q \leq r \leq s \leq p+1$ and $n-2=4 p+d$, where $0 \leq d \leq 3$. Every connected subgraph of the graph $K_{p} \cup K_{q} \cup K_{r} \cup K_{s}$ contains at most $p+1$ vertices. Moreover, at most two connected subgraphs of $H_{n}$ (or $H_{n}^{\prime}$ ) of order at least $p+2$ contain a vertex of $K_{2}$ (or $2 K_{1}$ ). Thus, because the integer $w=n-3 p-6=p+d-4$ is non-negative for $p \geq 4$, the sequence of the form $(w, p+2, p+2, p+2)((p+2, p+2, p+2)$ for $w=0)$ is admissible and non-realizable in both $H_{n}$ and $H_{n}^{\prime}$ for $n \geq 18$. It is easy to verify that for every $n$ such that $6 \leq n \leq 17, n \notin\{7,8,11\}$ there exists an admissible sequence of the form $(w, p+2, p+2, p+2),(p+2, p+2, p+2),(w, p+1, p+1, p+1)$ or $(p+2, p+2, p+2)$ non-realizable in both $H_{n}$ and $H_{n}^{\prime}$. Moreover, $H_{n}$ and $H_{n}^{\prime}$ are 2-connected, $\sigma_{2}\left(H_{n}\right)=\sigma_{2}\left(H_{n}^{\prime}\right)=\left\lfloor\frac{n}{2}\right\rfloor$ if $n \equiv 0(\bmod 4)$ and $\sigma_{2}\left(H_{n}\right)=\sigma_{2}\left(H_{n}^{\prime}\right)=\left\lfloor\frac{n}{2}\right\rfloor+1$ otherwise.

To conclude this section note that the graphs $G_{5}$ and $H_{i}, H_{i}^{\prime}$ with $i=7,8,11$, are easily seen to be avd.

\section{Some Known Results}

The first result characterizing avd star-like trees was found by Barth, Baudon and Puech [1] and, independently, by Horňák and Woźniak [7].

Proposition 1 A star-like tree $S(2, a, b)$ is avd if and only if $a$ and $b$ are coprime. Moreover, each sequence that is admissible for, but not realizable in the graph $S(2, a, b)$, is of the form $(d, \ldots, d)$, where $a \equiv b \equiv 0(\bmod d)$ and $d>1$.

The next proposition was presented in [14].

Proposition 2 Let $G$ be the graph of order $n \geq 4$ obtained by taking a path $P=$ $x_{1}, \ldots, x_{n-1}$, a single vertex $x$ and by adding the edges $x x_{i_{1}}, \ldots, x x_{i_{p}}$, where $1<$ $i_{1}<\cdots<i_{p}<n-1$ and $p \geq 1$. Then $G$ is not avd if and only if there are integers $d>1, \lambda, \lambda_{1}, \lambda_{2}, \ldots, \lambda_{p}$ such that $n=\lambda d$ and $i_{j}=\lambda_{j} d$ for $j=1, \ldots, p$. Moreover, each sequence that is admissible for, but not realizable in $G$, is of the form $\left(d^{\prime}, \ldots, d^{\prime}\right)$, where $i_{j} \equiv n \equiv 0\left(\bmod d^{\prime}\right), j=1, \ldots, p$, and $d^{\prime}>1$. 
Kalinowski et al. [10] characterized avd suns with at most three rays. We shall need the "two rays characterization".

Theorem 3 A graph $\operatorname{Sun}(a, b)$ is avd if and only if either its order $n$ is odd or both a and $b$ are even. Moreover, each sequence that is admissible for, but not realizable in the graph $\operatorname{Sun}(a, b)$ is of the form $(2, \ldots, 2)$.

In the proofs of the main results of this paper we will apply two generalizations of Ore's theorem [16]. The first one is due to Pósa [17].

Theorem 4 Let $G$ be a connected graph of order $n \geq 3$ such that

$$
\sigma_{2}(G) \geq d
$$

If $d<n$ then $G$ contains a path of length $d$ and if $d \geq n$, then $G$ is hamiltonian.

The second one was found by Bermond [3] and, independently, by Linial [12].

Theorem 5 Let $G$ be a 2-connected graph such that

$$
\sigma_{2}(G) \geq d
$$

Then $G$ contains either a cycle of length at least $d$ or a hamiltonian cycle.

Notice that any realization of the sequence $(2, \ldots, 2)$ is a perfect matching and that of the sequence $(2, \ldots, 2,1)$ is a quasi-perfect matching (of the involved graph). Therefore, the independence number $\alpha(G)$ of an $n$-vertex avd graph $G$ satisfies $\alpha(G) \leq$ $\lceil n / 2\rceil$.

In [15] the following was proved.

Theorem 6 Let $G$ be a connected graph of order $n$ such that $\sigma_{2}(G) \geq n-3, \alpha(G)$ is at most $\lceil n / 2\rceil$ and $G$ is isomorphic neither to $G_{6}$ nor to $G_{7}$. Then $G$ is avd.

\section{Preparatory Lemmas}

Lemma 7 Let $G$ be a connected graph of order $n, P=x_{1}, \ldots, x_{s}$ with $s \leq n-2 a$ longest path in $G$ and $Q=w_{1}, \ldots, w_{t}$ with $t \geq 2$ a longest path in $G[V(G) \backslash V(P)]$. Then

(i) $d_{P}\left(w_{1}\right)+d_{P}\left(w_{t}\right) \leq \max \{0,2\lceil(s-2 t) / 4\rceil\} \leq(s-t) / 2$ if $t \geq 3$;

(ii) $d_{P}\left(w_{1}\right)+d_{P}\left(w_{t}\right) \leq \max \{0,2\lceil(s-4) / 3\rceil\} \leq 2(s-2) / 3$ if $t=2$.

Proof Suppose first that $t \geq 3$ and observe that $N_{P}\left(w_{i}\right) \cap\left\{x_{1}, \ldots, x_{t}\right\}=\varnothing$ and $N_{P}\left(w_{i}\right) \cap\left\{x_{s-t+1}, \ldots, x_{s}\right\}=\emptyset$ for $i=1, t$, for otherwise $G$ has a path of order at least $s+1$, a contradiction. Thus, if $s-2 t \leq 0$, then $d_{P}\left(w_{1}\right)+d_{P}\left(w_{t}\right)=0$ and the inequalities in $(i)$ hold. Therefore, we may assume that $s-2 t \geq 1$. Since $P$ is 
a longest path in $G$, we have $P(i, j):=\left|N_{P}\left(w_{i}\right) \cap\left\{x_{j}, x_{j+1}, x_{j+2}, x_{j+3}\right\}\right| \leq 2$ and $P(1, j)+P(t, j) \leq 2$ for any $i \in\{1, t\}$ and $j \in\{t+1, \ldots, s-t\}$. It follows that

$$
\begin{aligned}
d_{P}\left(w_{1}\right)+d_{P}\left(w_{t}\right) & =\sum_{k=1}^{\lceil(s-2 t) / 4\rceil}(P(1, t+1+4(k-1))+P(t, t+1+4(k-1))) \\
& \leq 2\left\lceil\frac{s-2 t}{4}\right\rceil
\end{aligned}
$$

and the inequalities in (i) hold. A similar argument shows that (ii) is also true.

Lemma 8 Let $G$ be a connected graph of order $n, C=x_{1}, x_{2}, \ldots, x_{s}, x_{1}$ with $s \leq$ $n-2$ a longest cycle in $G$ and $Q=w_{1}, \ldots, w_{t}$ with $s>t \geq 2$ a longest path in $G[V(G) \backslash V(C)]$ with both endvertices having a neighbor in $C$. Then

(i) $d_{C}\left(w_{1}\right)+d_{C}\left(w_{t}\right) \leq 2\lceil(s-t) / 4\rceil \leq s / 2$ if $t \geq 3$;

(ii) $d_{C}\left(w_{1}\right)+d_{C}\left(w_{t}\right) \leq 2\lceil(s-2) / 3\rceil \leq 2 s / 3$ if $t=2$.

Proof Suppose $t \geq 3$. Since, by assumption, there are integers $i, j \in\{1,2, \ldots, s\}$ such that $x_{i} \in N_{C}\left(w_{1}\right)$ and $x_{j} \in N_{C}\left(w_{t}\right)$, it follows that for some $r \in\{1,2, \ldots, s\}$, $x_{r+1}, x_{r+2}, \ldots, x_{r+t} \notin\left(N_{C}\left(w_{1}\right) \cup N_{C}\left(w_{t}\right)\right)$. Using the same argument as in the proof of Lemma 7 we can show that (i) is true. The proof of (ii) is analogous.

Lemma 9 Let $G$ be a connected nontraceable graph of order $n$ with $\sigma_{2}(G) \geq n-k$ for some $k<(n+11) / 6$. If $P=x_{1}, x_{2}, \ldots, x_{s}$ is a longest path in $G$, then $S=$ $V(G) \backslash V(P)$ is independent.

Proof Suppose $S$ is not independent and let $H=G[S]$. Then $|V(H)| \geq 2$. Since $G$ is connected and $P$ a longest path, $x_{1}$ and $x_{s}$ are not adjacent. Moreover, by the classical Ore-type argument we have $d\left(x_{1}\right)+d\left(x_{s}\right) \leq s-1$, because otherwise $G$ would have a cycle containing all the vertices of $P$ and also a path of order at least $s+1$. Assume $d\left(x_{1}\right) \leq d\left(x_{s}\right)$. Hence $d\left(x_{1}\right) \leq(s-1) / 2$ and if $w$ is a vertex of $H$, then

$$
d(w) \geq n-k-(s-1) / 2,
$$

because $x_{1}$ and $w$ are not adjacent.

Case 1: $\Delta(H)=1$. Let $x$ and $y$ be two adjacent vertices of $H$. Hence $d_{H}(x)=$ $d_{H}(y)=1$. Now, by (1) and Lemma 7 ,

$$
2(n-k)-(s-1) \leq d(x)+d(y) \leq \frac{2}{3}(s-2)+2 \leq \frac{2}{3}(n-1) .
$$

Since $2(n-k)-(s-1) \geq 2 n-2 k-(n-3)=n+3-2 k$, we have $n+3-2 k \leq(2 n-2) / 3$ and this implies $n+11 \leq 6 k$, a contradiction.

Case 2: $\Delta(H) \geq 2$. Consider a longest path $Q=w_{1}, \ldots, w_{t}$ in $H$ of order $t \geq 3$. Clearly, $d_{H}\left(w_{1}\right)+d_{H}\left(w_{t}\right) \leq 2(t-1)$. Theorem 4 implies that

$$
s \geq n-k+1
$$


By Lemma 7 we also have

$$
d_{P}\left(w_{1}\right)+d_{P}\left(w_{t}\right) \leq(s-t) / 2
$$

Combining (1), (2) and (3) we get

$$
\begin{aligned}
& 2(n-k)-(s-1) \leq d\left(w_{1}\right)+d\left(w_{t}\right)=d_{H}\left(w_{1}\right)+d_{H}\left(w_{t}\right)+d_{P}\left(w_{1}\right)+d_{P}\left(w_{t}\right) \\
& \quad \leq 2(t-1)+\frac{1}{2}(s-t)=\frac{1}{2} s+\frac{3}{2} t-2 \leq \frac{1}{2} s+\frac{3}{2}(n-s)-2=\frac{3}{2} n-s-2 \\
& \quad \leq \frac{3}{2} n-(n-k+1)-2=\frac{1}{2} n+k-3 .
\end{aligned}
$$

Now, since $2(n-k)-(s-1) \geq 2 n-2 k-(n-4)=n-2 k+4$, we have $n-2 k+4 \leq n / 2+k-3$, therefore $6 k \geq n+14$, a contradiction.

Lemma 10 Let $G$ be a connected graph of order $n$ with a longest path of order $n-1$. If $\sigma_{2}(G) \geq n-k$ for some $k<(n+6) / 4$, then there is a longest path $P$ of order $n-1$ and a vertex $w \notin V(P)$ with $d(w) \geq(n-k) / 2$.

Proof Let $P=x_{1}, x_{2}, \ldots, x_{n-1}$ be a longest path in $G$, and let $w \in V(G) \backslash V(P)$. Hence $x_{1}, x_{n-1} \notin N(w)$. Suppose $d(w)<(n-k) / 2$.

By the classical Ore-type argument we have $d\left(x_{1}\right)+d\left(x_{n-1}\right) \leq n-2$. Assume without loss of generality that $d\left(x_{1}\right) \leq d\left(x_{n-1}\right)$. Hence $d\left(x_{1}\right) \leq(n-2) / 2$ and

$$
d(w) \geq n-k-(n-2) / 2>(n-2) / 4,
$$

because $x_{1}$ and $w$ are not adjacent. Since $d(w)<(n-k) / 2$ and $\sigma_{2}(G) \geq n-k$, we may assume $x_{2}, x_{n-2} \notin N(w)$, for otherwise in $G$ there is a path $P^{\prime}$ of order $n-1$ either with $x_{1} \notin V\left(P^{\prime}\right)$ and $d\left(x_{1}\right)>n-k-(n-k) / 2=(n-k) / 2$ or with $x_{n-1} \notin V\left(P^{\prime}\right)$ and $d\left(x_{n-1}\right)>(n-k) / 2$.

If $x_{j} \in N(w)$, then $x_{j+1} \notin N\left(x_{1}\right)$ and $x_{j+1} \notin N(w)$, since $P$ is a longest path. Moreover, we may assume $x_{j+2} \notin N\left(x_{1}\right)$, for otherwise there is a longest path $P^{\prime}$ of order $n-1$ such that $x_{j+1} \notin V\left(P^{\prime}\right)$, but $d\left(x_{j+1}\right)>(n-k) / 2$, and we are done. Hence, $d\left(x_{1}\right) \leq(n-1)-1-2 d(w)=n-2 d(w)-2$. Thus, by (4), $d\left(x_{1}\right)+d(w) \leq$ $n-d(w)-2<n-(n-2) / 4-2=(3 n-6) / 4$. But $(3 n-6) / 4<n-k$ for $k<(n+6) / 4$, a contradiction.

Lemma 11 Let $G$ be a connected graph of order $n \geq 15$ with a longest path $P$ of order $n-1$ and a vertex $w \notin V(P)$. If

$$
d(w) \geq \frac{n-5}{2}
$$

then $G$ is avd or $n$ is even and $G$ has no perfect matching. 
Proof Let $P=x_{1}, x_{2}, \ldots, x_{n-1}$ be a longest path in $G$, and let $w \in V(G) \backslash V(P)$. Suppose $G$ is not avd.

Since $G$ is nontraceable and $d(w) \geq \frac{n-5}{2} \geq \frac{n}{3}$ for $n \geq 15$, there is an index $a$ such that $x_{a} w \in E(G)$ and $x_{a+2} w \in E(G)$. By Proposition 2 there are integers $d>1$, $\lambda, \lambda^{\prime}$ and $\mu$ such that $a=\lambda d, a+2=\lambda^{\prime} d$ and $n=\mu d$, so $d=2, n$ is even and $(2, \ldots, 2)$ is the sequence not realizable in $G$. Thus $G$ has no perfect matching.

\section{2-Connected avd Graphs}

Theorem 12 Let $G$ be a 2-connected graph of order $n$ such that $G \notin\left\{H_{9}, H_{10}, H_{9}^{\prime}\right.$, $H_{10}^{\prime}$. If $G$ admits a perfect matching or a quasi-perfect matching and

$$
\sigma_{2}(G) \geq n-4
$$

then $G$ is avd.

Proof By Theorem 5, $G$ contains a cycle of length at least $n-4$. If $G$ has a cycle of length at least $n-1$, then $G$ is traceable, so also avd. Moreover, if $n \leq 7$ it follows that $\sigma_{2}(G) \geq 4 \geq n-3$ and Theorem 6 can be applied. Therefore, we shall assume that $G$ contains neither $C_{n}$ nor $C_{n-1}$ and $n \geq 8$. Suppose, contrary to our claim, that $G$ is not avd.

Case 1: $G$ has no cycle of length at least $n-3$, i.e., the circumference of $G$ equals $n-4$. Denote by $C$ a cycle of length $n-4$ with a given orientation and let $X:=$ $V(G) \backslash V(C)=\{x, y, z, t\}$.

Case 1.1: The set $X$ is independent. Suppose without loss of generality $d(x) \geq d(y)$ and let $A=N(x)=N_{C}(x)$. Since $x$ and $y$ are not adjacent and $d(x) \geq d(y)$, we have $d(x) \geq(n-4) / 2$. Note that no two neighbors of $x$ are consecutive on $C$, for otherwise $G$ would contain a cycle of length $n-3$. Hence $d(x)=(n-4) / 2, n$ is even and, because $x$ and $y$ are not adjacent, we have $d(y)=(n-4) / 2$. We can show in a similar way that $d(t)=d(z)=(n-4) / 2$. If $u$ and $v$ belong to $N(x)$, then $u^{+} v^{+} \notin E(G)$, because otherwise $u^{+}, v^{+}, v^{+2}, \ldots, u^{-}, u, x, v, v^{-}, v^{-2}, \ldots, u^{+}$would be a cycle of length $n-3$ contradicting our assumption. Consider a vertex $w \in\{y, z, t\}$. We have either $u^{+} w \notin E(G)$ or $v^{+} w \notin E(G)$, for otherwise replacing the subpath $u, u^{+}, \ldots, v, v^{+}$ of $C$ by the path $u, x, v, v^{-}, \ldots, u^{+}, w, v^{+}$we would obtain a cycle in $G$ of length $n-2$, a contradiction. Thus, $\left|N(w) \cap A^{+}\right| \leq 1$. Clearly, $N(w) \in\left\{A, A^{+}\right\}$. However, from $d(w)=(n-4) / 2 \geq 2$ it follows that $N(w)=A$.

Hence, $A^{+} \cup X$ is an independent set of cardinality $(n-4) / 2+4=(n+4) / 2>$ $\lceil n / 2\rceil$, a contradiction.

Case 1.2: The length of a longest path in $G[X]$ is one. We may assume without loss of generality that $t z \in E(G)$. Because $G$ is 2-connected there are two independent edges, say $t u$ and $z v$, joining the set $\{z, t\}$ to $C$. Clearly, the distance (on $C$ ) between $u$ and $v$ is at least three. Thus, there are at least four vertices of $C$ that do not belong to $N(z) \cup N(t)$, so $n \geq 10$. If $x y \notin E(G)$, then $d(x)=d(y)=(n-4) / 2$ (see Case 
$1.1)$, hence $d(z) \geq(n-4) / 2$ and $d(t) \geq(n-4) / 2$. By Lemma 8 ,

$$
n-4 \leq d(t)+d(z) \leq \frac{2(n-4)}{3}+2,
$$

thus $n \leq 10$. Hence $G$ has 10 vertices, $v=u^{+3}$ and $u^{+}, u^{-} \in N(x)$ or $v^{+}, v^{-} \in N(x)$. It follows that $G$ has a cycle of length at least $n-3$, a contradiction.

We may assume $x y \in E(G), d(z) \geq(n-4) / 2$ and $d(t) \geq(n-4) / 2$ (otherwise $d(x) \geq(n-4) / 2$ and $d(y) \geq(n-4) / 2)$. It follows that (5) holds and $n=10$.

Hence, $v=u^{+3}=u^{-3}, d_{C}(x)=d_{C}(y)=d_{C}(z)=d_{C}(t)=2, N_{C}(x)=$ $N_{C}(y)=N_{C}(z)=N_{C}(t)=\left\{u, u^{+3}\right\}$ and there are no edges between the sets $\left\{u^{+}, u^{+2}\right\}$ and $\left\{u^{+4}, u^{+5}\right\}$ (because the circumference of $G$ is 6). Thus, because $d\left(u^{+i}\right) \geq 3$, for $i=1,2,4,5$, we have $N\left(u^{+i}\right) \supset\left\{u, u^{+3}\right\}, i=1,2,4,5$ and $G$ is isomorphic to $H_{10}^{\prime}$ (if $u$ and $u^{+3}$ are not adjacent), or to $H_{10}$ (if $u$ and $u^{+3}$ are adjacent), which contradicts our assumption.

Case 1.3: The length of a longest path in $G[X]$ is two. We may assume that $P=x, y, z$ is a longest path in the graph induced by $X$.

Case 1.3.1: $d(t)=d_{C}(t)$. Because $G$ is 2-connected, $P$ can be chosen so that there are two independent edges, say $x u$ and $z v$, joining the ends of $P$ to $C$. Hence $n \geq 12$.

Applying a similar argument as in Case 1.1 we can easily show that $d(x) \geq(n-4) / 2$ and $d(z) \geq(n-4) / 2$. By Lemma 8 ,

$$
n-4 \leq d(x)+d(z) \leq \frac{n-4}{2}+4
$$

so $n \leq 12$. Therefore, $n=12, v=u^{+4}, N_{C}(x)=N_{C}(z)=\{u, v\}, d(x)=d(z)=$ $d(t)=4,\{u, v\} \subset N(t)$ (otherwise $G$ has a cycle of length $n-3$ ), so $u^{+2}, v^{+2} \in N(t)$ and it is easy to show that $G$ possesses a cycle of order $n-2$, a contradiction.

Case 1.3.2: $d(t)=d_{C}(t)+1$, i.e., $t$ and $y$ are adjacent and $X$ induces the graph $K_{1,3}$. Suppose there exists a vertex $u$ with $N_{C}(x)=N_{C}(z)=N_{C}(t)=\{u\}$. Thus, because $x z \notin E(G), d(x)+d(z)=2+2 \geq n-4$, so $n=8$, and, since $G$ is 2-connected, there is $v \in V(C), v \neq u$, such that $y v \in E(G)$, so $G$ contains a cycle of length at least 5 , a contradiction. Therefore, we may assume that there are two independent edges joining the ends of $P$ to $C$ and $n \geq 12$. Observe that there are at least two vertices $u, u^{\prime} \in\{x, z, t\}$ of degree at least $(n-4) / 2 \geq 4$, so the analogue of the inequalities (6), obtained when replacing $x$ by $u$ and $z$ by $u^{\prime}$, is true. Hence $n=12$ and $d_{C}(w) \geq(n-4) / 2-1=3$ for $w=u, u^{\prime}$, thus $G$ contains a cycle of length at least $n-3$, a contradiction.

Case 1.4: The subgraph induced by $X$ contains a path $P$ of length three. We may assume that $P=x, y, z, t$. If $x$ or $t$ has a neighbor on $C$, then $G$ is traceable, a contradiction. If $N_{C}(x)=N_{C}(t)=\emptyset$, then, because $d(x) \geq 2$ and $d(t) \geq 2, G[X]$ has a hamiltonian cycle. Clearly, there is an edge joining $X$ and $C$, so $G$ is traceable, again a contradiction.

Case 2: The circumference of $G$ is $n-3$. Let $C$ be a cycle of length $n-3 \geq 5$ and let $X=V(G) \backslash V(C)=\{x, y, z\}$. 
Case 2.1: $X$ is an independent set in $G$. Assume without loss of generality that $d(x) \geq$ $d(y) \geq d(z)$. Set $A=N(x)$. Since $x$ and $y$ are not adjacent and $d(x) \geq d(y)$, we have $d(x) \geq(n-4) / 2$. If $n$ is odd, then $d(x) \geq(n-3) / 2$, and, because no two neighbors of $x$ are consecutive on $C, d(x)=(n-3) / 2$. Now, applying the same argument as in Case 1.1 we can show that the set $A^{+} \cup\{x\}$ is independent and $\left|N(y) \cap A^{+}\right| \leq 1$. Suppose $\left|N(y) \cap A^{+}\right|=1$. Thus there are two different vertices $u$ and $v$ with $u \in A \cap N(y)$ and $v \in A^{+} \cap N(y)$. Then, because $v^{+} \neq u, v^{-} \neq u$ and $u^{-2} \in A$, the cycle $v^{+}, v, y, u, u^{+} \ldots, v^{-}, x, u^{-2}, u^{-3}, \ldots, v^{+}$has length $n-2$, and we obtain a contradiction. Finally, we conclude that $N(y) \subset A$. Now, using the same argument as above, we can show that $N(z) \subset A$ and $A^{+} \cup\{x, y, z\}$ is an independent set of cardinality $(n-3) / 2+3>\lceil n / 2\rceil$, so $G$ has no quasi-perfect matching and we get a contradiction.

Therefore, we can assume that $n$ is even and $d(x)=d(y)=d(z)=(n-4) / 2 \geq 2$. Consequently, any two consecutive neighbors of $x$ on $C$, except exactly one pair, are separated by one vertex of $C$, and this exceptional pair is separated by two vertices of $C$. Obviously, the sets $N(y)$ and $N(z)$ have an analogous property. Moreover, we can show as in Case 1.1 that the set $A^{+} \cup\{x\}$ is independent, $\left|N(y) \cap A^{+}\right| \leq 1$ and $\left|N(z) \cap A^{+}\right| \leq 1$. Clearly, $z$ or $y$ is adjacent to a vertex $v$ of $A^{+}$, for otherwise $A^{+} \cup X$ would be an independent set of cardinality $(n-4) / 2+3>\lceil n / 2\rceil$. Suppose $v \in A^{+} \cap N(y)$ and $v^{+} \in A$. Then, because $v^{+}$and $v^{+2}$ do not belong to $N(y)$, we have $v^{+3} \in N(y)$ and $v^{-}, x, v^{+}, v, y, v^{+3}, v^{+4}, \ldots, v^{-}$is a cycle of length $n-2$, a contradiction. Similarly, if $v \in A^{+} \cap N(z)$ and $v^{+} \in A$ then $G$ has a $C_{n-2}$, a contradiction. Assume then $v \in A^{+} \cap N(y)$ and $v^{+2} \in A$. It follows that $v^{-2} \notin N(y)$ and $v^{-3} \in A \cap N(y)$. Set $B=N(y)$ and consider the set $B^{+}=\left(A^{+} \backslash\{v\}\right) \cup\left\{v^{+}\right\}=A^{-}$ and suppose there is a vertex $u$ such that $u \in B^{+} \cap N(z)$. If $u^{+} \in B$ then we can show as above that $G$ has a $C_{n-2}$ and we get a contradiction. Suppose then $u^{+2} \in B$. Clearly, $u=v^{-2} \in A^{+}$and $u^{+} \in A$, which is impossible. It follows that $B=N(z)$, so $B^{+} \cup X$ is an independent set of cardinality $(n-4) / 2+3>n / 2=\lceil n / 2\rceil$, a contradiction.

Case 2.2: The set $X$ induces the disjoint union $K_{2} \cup K_{1}$. We may assume without loss of generality that $x y \in E(G), x z \notin E(G), y z \notin E(G)$ and $d(z) \leq(n-3) / 2$. Since $G$ is 2-connected, there are independent edges, say $x u$ and $y v$, connecting $\{x, y\}$ with $C$. This implies $n \geq 9$.

Suppose first $d(z)=\frac{n-3}{2}$ and $n \geq 9$ is odd. Using a similar argument as in Case 1.1 we can show that $N_{C}(x) \subset N(z)$ and $N_{C}(y) \subset N(z)$. Therefore, $u^{+2}$ and $v^{+2}$ both belong to $N(z)$, so $v^{+2}, z, u^{+2}, u^{+3}, \ldots, v, y, x, u, u^{-}, \ldots, v^{+2}$ is a cycle of length $n-2$, a contradiction.

Consider now the case $d(z)=\frac{n-4}{2}$ and $n$ is even. Hence $d(x) \geq(n-4) / 2$ and $d(y) \geq(n-4) / 2$. By Lemma 8 ,

$$
d(x)+d(y) \leq 2 \frac{n-3}{3}+2 \leq \frac{2 n}{3},
$$

therefore, $n \leq 12$. If $n=12$, we have $|V(C)|=9, N_{C}(x)=N_{C}(y)=\left\{u, u^{+3}, u^{+6}\right\}$ and $d(z)=4$. It follows that $w^{-}, w^{+} \in N(z)$ for some $w \in N_{C}(x)$ and $G$ has a cycle of length at least $n-2$, a contradiction. Suppose $n=10$. 
Now $d(x)=d(y)=d(z)=3$ and we may assume $N_{C}(x)=N_{C}(y)=\left\{u, u^{+3}\right\}$. Since $G$ does not contain a cycle of order at least 8 , we have $N(z)=\left\{u, u^{+3}, u^{+5}\right\}$, and the path $u^{+}, u^{+2}, u^{+3}, z, u^{+5}, u^{+6}, u, y, x$ together with the edge $u^{+3} u^{+4}$ form a spanning subgraph of $G$ which is isomorphic to the avd graph $S(2,3,7)$, a contradiction.

It remains the case $d(z) \leq \frac{n-5}{2}$. But now $d(x)+d(y) \geq n-3$, and, by (7), $n=9$. It follows that $d(z)=2, N_{C}(x)=N_{C}(y)=\left\{u, u^{+3}\right\}$, so $N(z) \cap\left\{u, u^{+3}\right\} \neq \emptyset$ since otherwise $G$ has a cycle of order at least $n-2$. Suppose $N(z)=\left\{u, u^{+3}\right\}$. We have $d\left(u^{+i}\right)=3$, and so $N\left(u^{+i}\right) \supset\left\{u, u^{+3}\right\}, i=1,2$, 4, 5. If $u u^{+3} \in E(G)$, then $G$ is isomorphic to $H_{9}$, and if $u u^{+3} \notin E(G)$, we obtain $H_{9}^{\prime}$, a contradiction. Assume then $\left|N(z) \cap\left\{u, u^{+3}\right\}\right|=1$, say $u, u^{+2} \in N(z)$. Now the path $x, y, u^{+3}, u^{+4}, u^{+5}, u, z$, $u^{+2}, u^{+}$is a hamiltonian path in $G$, a contradiction.

Case 2.3: The set $X$ induces a connected subgraph $H$ of $G$. Then $H$ contains a path of order three, and, because at least two vertices of $X$ are joined to $G-X$ (since $G$ is 2-connected), $G$ is traceable, a contradiction.

Case 3: $G$ contains a cycle of length $n-2$ (i.e., the circumference of $G$ is $n-2$ ). Let $C$ be such a cycle with a given orientation and let $x$ and $y$ be the two vertices of $G$ outside $C$. Since $G$ is 2-connected, these two vertices together with $C$ and two independent edges connecting $\{x, y\}$ with $C$ form a spanning subgraph $H$ of $G$ isomorphic to a graph $\operatorname{Sun}(a, b)$. By our assumption $G$ is not avd, so it follows from Theorem 3 that $n$ is even, $a$ is odd and $G$ has no perfect matching, a final contradiction.

\section{Connected avd Graphs}

Theorem 13 Let $G$ be a connected graph of order $n \geq 11$. If $G$ admits a perfect matching or a quasi-perfect matching and

$$
\sigma_{2}(G) \geq n-4
$$

then $G$ is avd.

Proof Suppose $G$ is not avd. Therefore, $G$ is not traceable and, by Theorem 12, $G$ is not 2-connected. Let $x$ denote a cut vertex of $G$ and let $A_{1}, A_{2} \ldots, A_{s}$ be the connected components of $G-x$. Clearly, $s=2$, since otherwise $\sigma_{2}(G) \leq \frac{2(n-1)}{3}$, a contradiction because $n \geq 11$. Put $n_{i}=\left|V\left(A_{i}\right)\right|, i=1,2$. If $n_{1} \leq n-6$ and $n_{2} \leq n-6$, then $d_{A_{i}}(x)+d_{A_{i}}(y) \geq n_{i}$ for each pair $x, y$ of nonadjacent vertices of $A_{i}, i=1,2$, thus every component $A_{i}$ is either hamiltonian, or isomorphic to $K_{2}$, or else isomorphic to $K_{1}$. Thus $G$ is traceable, a contradiction.

Suppose that $n_{1} \geq n-5$. Then $n_{2} \leq 4 \leq n-6$ and there is a hamiltonian path in the graph induced by $\{x\} \cup V\left(A_{2}\right)$ that starts at $x$. Thus, there is a vertex $y \in V\left(A_{1}\right)$ with $d(y) \leq\left(n_{1}+1\right) / 2$, because otherwise there is a hamiltonian cycle in $A_{1}$ and $G$ is traceable, a contradiction. Now, if $u$ is a vertex of $A_{2}$, then $n-4 \leq d(y)+d(u) \leq$ $\left(n_{1}+1\right) / 2+n_{2}=\left(n+n_{2}\right) / 2$. Hence $n \leq 8+n_{2} \leq 12$ and $n_{2} \in\{3,4\}$. By considering a longest path in $G\left[V\left(A_{1}\right) \cup\{x\}\right]$ starting at $x$ we can easily show that $G$ is traceable, a final contradiction. 
The next theorem concerns the case where $\sigma_{2} \geq n-5$. In order to avoid many exceptional graphs of small order, we restrict our attention to graphs of order $n \geq 20$. This will allow us, in particular, to make use of Lemma 9.

Theorem 14 Let $G$ be a connected graph of order $n \geq 20$. If $G$ admits a perfect matching or a quasi-perfect matching and

$$
\sigma_{2}(G) \geq n-5
$$

then $G$ is avd.

Proof Suppose $G$ is not avd. Thus $G$ is not traceable. Let $P=x_{1}, \ldots, x_{n-p}$ be a longest path in $G$. By Theorem 4 we have $1 \leq p \leq 4$. Let $X=V(G) \backslash V(P)=$ $\left\{w_{1}, \ldots, w_{p}\right\}$. Since $k=5<(n+11) / 6$ for $n \geq 20$, by Lemma 9 the set $X$ is independent. Thus, there are four cases to be considered.

Case 1: $p=1$.

Thus $X=\{w\}$. Since $k=5<(n+6) / 4$ for $n \geq 20$, it follows from Lemma 10 that there is a path $P^{\prime}$ of order $n-1$ and a vertex $u \notin V\left(P^{\prime}\right)$ with $d(u) \geq(n-5) / 2$. By Lemma 11, $n$ is even and $G$ has no perfect matching, a contradiction.

Case 2: $p=2$.

Then, the set $X$ consists of two independent vertices $w_{1}$ and $w_{2}$ with $d\left(w_{i}\right) \leq \frac{n-3}{2}$, $i=1,2$. Assuming $d\left(w_{1}\right) \geq d\left(w_{2}\right)$ we get

$$
\frac{n-5}{2} \leq d\left(w_{1}\right) \leq \frac{n-3}{2}
$$

Case 2.1: $n$ is even.

Then, by inequality (8), we have $d\left(w_{1}\right)=\frac{n-4}{2} \geq 8$. Let $A=N\left(w_{1}\right)$. Clearly, $\left|A^{+} \cap N\left(w_{2}\right)\right| \leq 1$. Suppose that $a^{+} \in N\left(w_{2}\right)$ for some $a \in A$. Thus, there is $b \in V(P)$ such that $\left\{b, b^{+2}, b^{+4}\right\} \subset A$ and $\left[a, a^{+}\right] \cap\left[b, b^{+4}\right]=\emptyset$. If $b$ succeeds $a^{+}$on $P$, then, since $d\left(w_{2}\right) \geq \frac{n-6}{2}$, there is $i \in\{2,4\}$ such that $b^{+i} \in N\left(w_{2}\right)$ and $x_{1}, x_{2}, \ldots, a, w_{1}, b^{+(i-2)}, \ldots, a^{+}, w_{2}, b^{+i}, \ldots, x_{n-2}$ is a path of order $n-1$, a contradiction. Otherwise, $a^{+}$succeeds $b$ on $P$ and we can find a path of order $n-1$ similarly as above, again a contradiction.

Thus $N\left(w_{2}\right) \subset A$ and by using a typical Ore-type argument, it is easy to see that the set

$$
B=A^{+} \cup\left\{x_{1}, w_{1}, w_{2}\right\}
$$

is independent. But $|B| \geq \frac{n-4}{2}+3=\frac{n+2}{2}>\left\lceil\frac{n}{2}\right\rceil$, which contradicts the existence of a perfect matching in $G$.

Case 2.2: $n$ is odd.

Consider first the case $d\left(w_{1}\right)=\frac{n-3}{2}$. We define the sets $A$ and $B$ as above, and by the same argument we prove the independence of $B$. Now we have $|B| \geq \frac{n-3}{2}+3=$ $\frac{n+3}{2}=\frac{n+1}{2}+1$, which contradicts the existence of a quasi-perfect matching in $G$. 
So, we may assume that $d\left(w_{1}\right)=\frac{n-5}{2}$. The condition $\sigma_{2}(G) \geq n-5$ also implies that $d\left(w_{2}\right)=\frac{n-5}{2}$. So, the vertices $w_{1}$ and $w_{2}$ have the same number of neighbors on $P$. We shall prove that in fact we have:

Claim $N\left(w_{1}\right)=N\left(w_{2}\right)$.

Let $A=N\left(w_{1}\right)$. Suppose first that there exists a vertex $a \in A$ such that $a^{+}$belongs to $N\left(w_{2}\right)$. Without loss of generality we may assume that the distance on $P$ between $a$ and $x_{n-2}$, the second end of $P$, is $>9$, since otherwise we could exchange $w_{1}$ with $w_{2}$ and take the reverse order on $P$ (recall that $n \geq 20$ ). Observe that the edge $w_{1} x_{n-3}$ does not belong to $E(G)$, since otherwise the path $x_{1}, x_{2}, \ldots, a, w_{1}, x_{n-3}, x_{n-4}, \ldots$, $a^{+}, w_{2}$ would be longer than $P$, a contradiction. Consider now the edge $w_{1} x_{n-4}$. If it belongs to $E(G)$, then the path $x_{1}, x_{2}, \ldots, a, w_{1}, x_{n-4}, x_{n-5}, \ldots, a^{+}, w_{2}$ would be a longest path in $G$ with two adjacent vertices outside of it. This would be a contradiction with Lemma 9. Since two elements of $N\left(w_{1}\right)$ cannot be consecutive on $P$ and neither $w_{1} x_{n-3}$ nor $w_{1} x_{n-4}$ belongs to $E(G)$, a simple counting argument shows that $w_{1} x_{n-5} \in E(G), w_{1} x_{2} \in E(G)$ and the distance on $P$ between consecutive vertices of $A$ (with respect to $P$ ) is exactly two. But then $a^{+2} \in A$ and the path $x_{n-2}, x_{n-3}, \ldots, a^{+2}, w_{1}, x_{2}, x_{3}, \ldots, a^{+}, w_{2}$ is longer than $P$, a contradiction. Thus $a^{+} \notin N\left(w_{2}\right)$ and, by a similar argument, $a^{-} \notin N\left(w_{2}\right)$.

Finally, consider the case where $a^{+2} \in N\left(w_{2}\right) \backslash A$. We may assume $a^{+}, a^{+3} \notin A$, so there exists a vertex $y\left(=a^{+2}\right)$, a neighbor of $w_{2}$, such that the segment $\left[y^{-}, y^{+}\right]$is disjoint with $A$. Moreover, such a segment is exactly one, so we may assume $w_{1} x_{2} \in$ $E(G)$. It is easy to see that if $y^{-} y^{+} \notin E(G)$, then the set

$$
B=A^{+} \cup\left\{x_{1}, y^{+}, w_{1}, w_{2}\right\}
$$

is independent. But $|B|=\frac{n-5}{2}+4=\frac{n+3}{2}>\left\lceil\frac{n}{2}\right\rceil$, which contradicts the existence of a quasi-perfect matching in $G$.

On the other hand, if $y^{-} y^{+} \in E(G)$, then the path

$$
x_{1}, x_{2}, \ldots, y^{-2}, w_{1}, x_{n-3}, x_{n-4}, y^{+}, y^{-}, y, w_{2}
$$

is longer than $P$ (the case where $y=x_{n-3}$ is easy to check). This finishes the proof of the claim.

Denote by $a_{1}, a_{2}, \ldots, a_{r}, r=\frac{n-5}{2}$, the consecutive (with respect to the natural orientation of $P$ ) elements of $A$. The set $V(P) \backslash A$ is clearly the union of segments $\left[x_{1}, a_{1}^{-}\right],\left[a_{r}^{+}, x_{n-2}\right]$ and those of the form $\left[a_{i}^{+}, a_{i+1}^{-}\right]$. By a simple counting argument it is easy to show these segments contain one, two or three elements. Moreover, we have either exactly one segment containing three elements or exactly two segments containing two elements (while the remaining segments contain exactly one element). In this way, the proof of the Case 2.2 splits naturally into two subcases.

Case 2.2.1: There is one three-element segment.

Denote by $u_{1}, u_{2}, u_{3}$ the consecutive elements of this segment. Without loss of generality we may assume that $x_{2} \in A$. Let $\tau=\left(\alpha_{1}, \alpha_{2}, \ldots, \alpha_{k}\right), \alpha_{1} \geq \alpha_{2} \geq \cdots \geq \alpha_{k}$, be an admissible sequence for $G$. We shall show that $\tau$ is realizable in $G$. If $\alpha_{1} \geq 4$ then we define $V_{1}$ as the set containing the vertices $x_{1}, x_{2}, w_{1}, w_{2}$ as well as (for $\alpha_{1} \geq 5$ ) 
some consecutive vertices of $P$ with $x_{3}$ as the first one. The graph induced by $V_{1}$ is evidently connected and the remaining part of $G$ is traceable.

If $\alpha_{1}=3$ and $\alpha_{2}=3$, then we define $V_{1}$ as the set containing the vertices $\left\{x_{1}, x_{2}, w_{1}\right\}$ and $V_{2}$ as the set containing the vertices $\left\{u_{1}, u_{2}, u_{3}\right\}$. Now, the graph induced by $V_{1}$ is a star, the graph induced by $V_{2}$ is a path (or a cycle) on three vertices and the remaining part of $G$ is traceable.

Since the sequence $(3,2, \ldots, 2)$ is realizable in $G$ by assumption of the theorem, we are done.

Case 2.2.2: There are two two-element segments.

As above, let $\tau=\left(\alpha_{1}, \cdots, \alpha_{k}\right), \alpha_{1} \geq \alpha_{2} \geq \ldots \geq \alpha_{k}$, be an admissible sequence for $G$. We shall show that $\tau$ is realizable in $G$.

If $\alpha_{1} \geq 4$ then we proceed as above except for the case where $\alpha_{1}=4$ and both "large" segments are on the ends of $P$. In this case we define $V_{1}$ as the set containing the vertices $\left\{x_{1}, x_{2}, x_{3}, w_{1}\right\}$. Evidently, the graph induced by $V_{1}$ is connected and it is easy to see that the remaining part of $G$ has a spanning subgraph isomorphic to the graph $S(2,2, n-6)$, which is avd by Proposition 1 .

If $\alpha_{1}=3$ then we define $V_{1}$ as follows: $V_{1}=\left\{a_{j+1}^{-}, a_{j+1}, a_{j+1}^{+}\right\}$where $a_{j}, a_{j+1}$, $a_{j+2}$ are three consecutive vertices of $A$ such that both segments $\left[a_{j}^{+}, a_{j+1}^{-}\right]$and $\left[a_{j+1}^{+}, a_{j+2}^{-}\right]$contain one element. Such vertices exist since $n \geq 20$. Again, it is easy to see that the remaining part of the graph has a spanning subgraph which is avd by Proposition 2.

Since all other possible admissible sequences are evidently realizable in $G$, this finishes the proof of this case.

Case 3: $p=3$.

Then, the set $X$ consists of three independent vertices $w_{1}, w_{2}$ and $w_{3}$. Let $d\left(w_{1}\right) \geq$ $d\left(w_{2}\right) \geq d\left(w_{3}\right)$. Then, by assumption on $\sigma_{2}(G), \frac{n-4}{2} \geq d\left(w_{1}\right) \geq \frac{n-5}{2}$.

Let $A=N\left(w_{1}\right)$. Using a similar argument as in Case 2.1, we can easily show that $N\left(w_{i}\right) \subset A$ for $i=2,3$. Thus the set

$$
B=A^{+} \cup\left\{x_{1}, w_{1}, w_{2}, w_{3}\right\}
$$

is independent. But $|B| \geq \frac{n-5}{2}+3=\frac{n+3}{2}>\left\lceil\frac{n}{2}\right\rceil$, which contradicts the existence of a perfect matching or a quasi-perfect matching in $G$.

Case 4: $p=4$.

The set $X$ consists of four independent vertices $w_{1}, w_{2}, w_{3}$ and $w_{4}$. Let $d\left(w_{1}\right) \geq$ $d\left(w_{2}\right) \geq d\left(w_{3}\right) \geq d\left(w_{4}\right)$. By assumption on $\sigma_{2}$, we have $d\left(w_{1}\right)=d\left(w_{2}\right)=d\left(w_{3}\right)=$ $d\left(w_{4}\right)=\frac{n-5}{2}$.

Let $A=N\left(w_{1}\right)$. Again, it is easy to see that the set

$$
B=A^{+} \cup\left\{x_{1}, w_{1}, w_{2}, w_{3}, w_{4}\right\}
$$

is independent. However, the inequality $|B| \geq \frac{n-5}{2}+5=\frac{n+5}{2}>\left\lceil\frac{n}{2}\right\rceil$ contradicts the existence of a perfect matching or a quasi-perfect matching in $G$.

This finishes the proof of the theorem. 


\section{A problem}

The requirement $\sigma_{2}(G) \geq n-5$ in Theorem 14 is probably not the best possible. We suggest to consider the following problem which is based on our results.

Problem Find a function $f(n)$ and a constant $n_{0}$ that have the following properties: if $G$ is a connected graph of order $n \geq n_{0}$ that admits a perfect matching or a quasi-perfect matching and fullfils

$$
\sigma_{2}(G) \geq f(n)
$$

then $G$ is avd.

The graphs $G_{n}$ show that such a function satisfies $f(n)>\sigma_{2}\left(G_{n}\right) \geq\left\lfloor\frac{2 n}{3}\right\rfloor-1$. By Theorem 14 we have $f(n) \leq n-6$ for $n \geq 20$. We believe that $f(n) \leq n-7$ for sufficiently large $n$. However, a proof of an analogue of Theorem 14 with $\sigma_{2}(G) \geq n-6$ following our ideas would be technical and long.

Open Access This article is distributed under the terms of the Creative Commons Attribution Noncommercial License which permits any noncommercial use, distribution, and reproduction in any medium, provided the original author(s) and source are credited.

\section{References}

1. Barth, D., Baudon, O., Puech, J.: Network sharing: a polynomial algorithm for tripods. Discrete Appl. Math. 119, 205-216 (2002)

2. Barth, D., Fournier, H.: A degree bound on decomposable trees. Discrete Math. 306, 469-477 (2006)

3. Bermond, J.C.: On Hamiltonian walks. In: Nash-Wiliams, C.St.J.A., Sheehan, J. (eds.) Proc. Fifth British Combinatorial Conference, Utilitas Math., pp. 41-51 Winnipeg, (1976)

4. Bondy, JA., Murty, USR.: Graph Theory with Applications. Elsevier North Holland, New York (1976)

5. Cichacz, S., Görlich, A., Marczyk, A., Przybyło, J., Woźniak, M.: Arbitrarily vertex decomposable caterpillars with four or five leaves. Discuss. Math. Graph Theory 26, 291-305 (2006)

6. Győri, E.: On division of graphs to connected subgraphs. In: Combinatorics. Proc. Fifth Hungarian Colloq., Keszthely, 1976, vol. I, pp. 485-494, Colloq. Math. Soc. János Bolyai, vol. 18, North-Holland, Amsterdam, New York (1978)

7. Horňák, M., Woźniak, M.: On arbitrarily vertex decomposable trees. Discrete Math. 308, 12681281 (2008)

8. Horňák, M., Woźniak, M.: Arbitrarily vertex decomposable trees are of maximum degree at most six. Opuscula Mathematica 23, 49-62 (2003)

9. Horňák, M., Tuza, Zs., Woźniak, M.: On-line arbitrarily vertex decomposable trees. Discrete Appl. Math. 155, 1420-1429 (2007)

10. Kalinowski, R., Pilśniak, M., Woźniak, M., Zioło, I.: Arbitrarily vertex decomposable suns with few rays. Discrete Math. 309, 3726-3732 (2009)

11. Kalinowski, R., Pilśniak, M., Woźniak, M., Zioło, I.: On-line arbitrarily vertex decomposable suns. Discrete Math. 309, 6328-6336 (2009)

12. Linial, N.: A lower bound for the circumference of a graph. Discrete Math. 15, 297-300 (1976)

13. Lovász, L.: A homology theory for spanning trees of a graph. Acta Math. Acad. Sci. Hungar. 30, 241251 (1977)

14. Marczyk, A.: A note on arbitrarily vertex decomposable graphs. Opuscula Math. 26, 109-118 (2006)

15. Marczyk, A.: An Ore-type condition for arbitrarily vertex decomposable graphs. Discrete Math. 309, 3588-3594 (2009)

16. Ore, O.: Note on hamilton circuits. Am. Math. Monthly 67, 55 (1960)

17. Pósa, L.: A theorem concerning Hamiltonian lines. Publ. Math. Inst. Hungar. Acad. Sci. 7, 225226 (1962) 